\title{
Death from airways obstruction: accuracy of certification in Northern Ireland
}

\author{
E T Smyth, S C Wright, A E Evans, D G Sinnamon, J MacMahon
}

\begin{abstract}
Background - Studies of mortality from asthma and chronic obstructive pulmonary disease (COPD) have relied on death certification or registration for case finding. The aim of this study was to determine the accuracy of death certification and registration in asthma and COPD.

Methods - All death certificates in Northern Ireland for 1987 where asthma or COPD (defined as International Classification of Diseases 9th Revision (ICD9) $490,491,492,496)$ were listed in part $I$ or part II were identified. The following certificates were then selected for further investigation: those mentioning asthma for all ages, those mentioning COPD for ages less than 56 years, and a $50 \%$ sample of those mentioning COPD aged 56-75 years. For these selected deaths the general practitioners' case notes, hospital records, and necropsy findings were reviewed. Questionnaires detailing the clinical history and circumstances of death were completed by the general practitioner by post and by a close relative or associate of the deceased (doctor administered) if, after initial investigation, the death was likely to be due to COPD or asthma. A panel of two respiratory physicians reviewed each death and, using clinical diagnostic criteria, assessed the accuracy of the registered cause of death.
\end{abstract}

Results - Of 50 registered asthma deaths 43 were confirmed as being due to asthma. In nine registered deaths from COPD in cases aged less than 56 years one was confirmed as COPD, two as asthma, and six as other respiratory conditions. Of 105 registered deaths from COPD in cases aged 56-75, 42 were confirmed as COPD, 27 as asthma, eight as other respiratory conditions, and 28 as other causes. Although few errors in registration were found, $21 \%$ of certificates mentioning asthma and $38 \%$ of certificates mentioning COPD but not asthma in part I were subject to variable application of the classification rules by the registering officers. For all deaths under 75 years of age in Northern Ireland in 1987 where either asthma or COPD was mentioned anywhere on the death certificate, the estimated sensitivity and specificity of the registered cause of death in predicting the "true" cause of death were $29 \%$ and $98.6 \%$ for asthma and $69 \%$ and $70 \%$ for COPD.

Conclusions - In a population of subjects where asthma or COPD was mentioned anywhere on the death certificate, the registered cause of death is a relatively poor indicator of the "true" cause of death for both asthma and COPD. Variation occurred in the application of death classification rules by registration officers. Many deaths certified and registered as COPD could have been called asthma using current standards of clinical diagnosis. In studies investigating risk factors for deaths from asthma, case finding should consider deaths registered as COPD.

(Thorax 1996;51:293-297)

Keywords: asthma, chronic obstructive pulmonary disease, death certification, Northern Ireland.

Recent reports have shown an increase in deaths from asthma in the older age groups. ${ }^{1}$ Whether this increase in deaths is due to a rise in asthma prevalence, an increase in case fatality, or changes in diagnostic fashion or death certification practice among doctors is unknown. Changes in the international death classification rules or application of these rules by registering officers may also lead to variation in mortality statistics. Accuracy of death certification is greatest in younger age groups and declines in older age groups. Inaccuracy is thought to be mainly due to diagnostic confusion between chronic asthma and chronic obstructive pulmonary disease (COPD) which can lead to arbitrary diagnostic coding and death certification. Studies investigating trends and risk factors in asthma death have concentrated on younger age groups. ${ }^{2-4}$ Since mortality appears to be rising in older age groups, a detailed investigation into the accuracy of death certification is necessary for a clearer interpretation of mortality trends.

In a previous study we have shown that, for the years 1981-4, approximately one third of registered asthma deaths in Northern Ireland did not die from asthma. ${ }^{5}$ A similar number of asthma deaths were registered under other diagnoses including COPD, but the study did not examine deaths registered as COPD in cases aged over 55 years. Little is known of the accuracy of death certification in COPD and to date no comprehensive examination of registered deaths mentioning all forms of COPD has been reported.

The purpose of our study was to investigate the accuracy of death certification and registration in asthma in all ages and COPD in ages up to 75 years. 
Methods

The study was approved by the Queen's University Belfast medical ethics committee, the four local medical committees representing the general practitioners in Northern Ireland as well as all relevant hospital medical staff committees.

All deaths registered in Northern Ireland during 1987 were identified and certificates mentioning asthma (International Classification of Disease 9th Revision (ICD9) 493) or COPD (ICD9 496 chronic airways obstruction not elsewhere classified; ICD9 491 chronic bronchitis; ICD9 492 emphysema; ICD9 490 bronchitis not specified as acute or chronic) in part I or part II were extracted. This includes all certificates where asthma or COPD was the registered cause of death but certificates were also extracted if any mention of asthma or COPD was made on the certificate whether or not asthma or COPD was the underlying cause of death.

Following extraction the following three groups of certificates were selected for further investigation: (1) all 103 certificates mentioning asthma anywhere on the certificate; (2) all 21 certificates mentioning COPD (defined above) but not asthma anywhere on the certificate of cases aged under 56 years; and (3) a sample of 226 deaths representing $50 \%$ of certificates mentioning COPD but not asthma anywhere on the certificate of cases aged 56-75. This sample, necessary because of the large numbers in this group, was chosen by listing all certificates by registration code (codes allocated by district and date of death) and using random numbers generated on a Casio $\mathrm{fx}-82 \mathrm{~B}$ calculator.

For the 350 deaths in total selected in the three groups described above the following were obtained: 340 (97\%) general practitioner case notes from the Northern Ireland Central Services Agency; summaries from all 25 necroscopies; and all available hospital records (329 deaths). Questionnaires detailing the diagnosis, clinical history, prescribed medication, and circumstances of death were sent to each general practitioner with the case notes, of which $249(71 \%)$ were returned completed. All the information was summarised by one of the authors (ETS) and presented to two respiratory physicians (JMacM and DGS) who were blinded to the diagnosis on the death certificate. They decided whether the patient had died from asthma, COPD, or "other". A decision could not be made in some cases and was deferred until further information was obtained.

The following criteria adapted from those developed by the American Thoracic Society ${ }^{6}$ were used to diagnose asthma: (1) a clear clinical history (the features considered were early age of onset, smoking history, variability of symptoms, nocturnal symptoms, and atopic history); (2) documented evidence of airways obstruction with $>20 \%$ reversibility; and (3) necroscopic evidence of hyperinflation and mucous plugging. At least one of these criteria was required and the circumstances of death had to be compatible. The diagnosis of COPD was based on exclusion of the above cases and documented evidence of significant airways obstruction or necroscopic evidence of chronic bronchitis or emphysema, together with compatible circumstances of death.

If death from asthma or COPD was likely, or if the panel was undecided, a doctor (ETS) administered questionnaire was completed by the closest relative or associate of the deceased. Of 210 interviews requested by the panel the general practitioner gave permission in 151 $(72 \%)$ cases and $138(66 \%)$ relatives or close associates agreed to an interview. This further information was presented to the panel which, using the above criteria, decided whether death was due to asthma, COPD, or "other". In five cases, although the patient died of airways obstruction, the panel remained undecided about the nature of the chest condition and these were included as deaths from COPD. Both before and after the interview of the relative a random selection of cases $(10 \%)$ was blindly resubmitted to the panel to assess the reproducibility of the diagnosis. The official registration ICD9 code for each death was obtained from the central Northern Ireland Register of Births, Deaths and Marriages and the accuracy of registration was determined by comparing the registered cause of death with the authors' assessment of the underlying cause of death from a copy of the certificate using the classification rules published by the World Health Organisation. ${ }^{7}$ A registration error was defined as a death registered in one of the three groups of asthma, COPD or "other" (defined as any death other than asthma or COPD) when the death should have been registered in one of the other two groups. No attempt was made to identify classification errors within groups.

\section{Results}

The results have been categorised according to whether the registered cause of death was asthma, COPD, or "other" (table). There were 50 registered deaths due to asthma (certificates mentioning asthma and registered as asthma using internationally agreed rules by the registering officers) and the panel agreed that asthma was the cause of death in $43(86 \%)$ of them. In the other seven cases the panel concluded that death was due to myocardial infarction (2), pneumonia (2), pneumothorax (1), carcinoma of stomach (1), and COPD (1). In one of the 50 certificates the rules of classification had been misapplied by the registering officer and the panel agreed with the amended underlying cause of death (pneumothorax).

When COPD was the registered cause of death the results were divided according to whether or not asthma was mentioned anywhere on the certificate. When asthma was not mentioned the results have also been divided by age group to allow for the sampling process (table). There were 10 certificates which mentioned asthma and the panel decided that death was due to asthma in seven of them, to COPD in two, and to "other" causes in one case 
Distribution of death certificate diagnosis, registered cause of death, and panel diagnosis

\begin{tabular}{|c|c|c|c|c|c|c|c|}
\hline \multirow{2}{*}{$\begin{array}{l}\text { Condition mentioned } \\
\text { on certificate }\end{array}$} & \multirow{2}{*}{$\begin{array}{l}\text { Registered } \\
\text { cause of death }\end{array}$} & \multirow{2}{*}{$\begin{array}{l}\text { Age range of } \\
\text { sample (years) }\end{array}$} & \multirow{2}{*}{$\begin{array}{l}\text { Sampling } \\
\text { fraction }\end{array}$} & \multirow{2}{*}{$\begin{array}{l}\text { No of } \\
\text { certificates }\end{array}$} & \multicolumn{3}{|c|}{ Panel diagnosis } \\
\hline & & & & & Asthma & $C O P D$ & "Other" \\
\hline Asthma & Asthma & $\underset{(<76)}{\text { All }}$ & All & $\begin{array}{c}50 \\
(47)\end{array}$ & $\begin{array}{c}43 \\
(41)\end{array}$ & $\begin{array}{c}1 \\
(1)\end{array}$ & $\begin{array}{c}6 \\
(5)\end{array}$ \\
\hline Asthma & COPD & $\begin{array}{l}\text { All } \\
(<76)\end{array}$ & All & $\begin{array}{l}10 \\
(10)\end{array}$ & $\begin{array}{c}7 \\
(7)\end{array}$ & $\begin{array}{c}2 \\
(2)\end{array}$ & $\begin{array}{l}1 \\
(1)\end{array}$ \\
\hline Asthma & Other & $\begin{array}{l}\text { All } \\
(<76)\end{array}$ & All & $\begin{array}{c}43 \\
(30)\end{array}$ & $\begin{array}{l}14 \\
(8)\end{array}$ & $\begin{array}{c}0 \\
(0)\end{array}$ & $\begin{array}{c}29 \\
(22)\end{array}$ \\
\hline COPD & COPD & $<56$ & All & 9 & 2 & 1 & 6 \\
\hline COPD & Other & $<56$ & All & 12 & 1 & 1 & 10 \\
\hline COPD & COPD & $56-75$ & $1: 2$ & 105 & 27 & 42 & 36 \\
\hline COPD & Other & $56-75$ & $1: 2$ & 121 & 14 & 19 & 88 \\
\hline Sample total & & & & 350 & 108 & 66 & 176 \\
\hline
\end{tabular}

Values in parentheses for ages $<76$ comprise a subgroup used for calculation of sensitivity and specificity in text.

(table). In three deaths confirmed as asthma "asthmatic bronchitis" had been incorrectly registered as COPD by the registering officer. In a further four confirmed asthma deaths "acute asthma due to" COAD or chronic bronchitis had been correctly registered as COPD although the use of both terms on the death certificate was clearly ambiguous. In nine deaths registered as COPD with no mention of asthma in cases aged less than 56 years the panel confirmed the diagnosis to be COPD in one, asthma in two, and "other" in six. There were no errors in registration. In 105 deaths registered as COPD with no mention of asthma in the 56-75 year age group the panel confirmed death to be due to COPD in $42(40 \%)$, asthma in $27(26 \%)$, and "other" in $36(34 \%)$; the other diagnoses were myocardial infarction (4), cardiac failure due to ischaemic heart disease (3), cerebrovascular accidents (2), lung carcinoma (2), other carcinoma (3), post tuberculous lung fibrosis (4), other fibrotic lung diseases (3), acute pulmonary embolism (1), miscellaneous (14). Out of these 105 deaths three errors in registration were found and the panel agreed with the amended diagnoses of carcinoma of lung, carcinoma of mouth, and post tuberculous lung fibrosis.

"Other" registered causes of death have been categorised by whether or not asthma or COPD was mentioned anywhere on the certificate. Certificates mentioning both conditions were included in the asthma group. When COPD was mentioned the results have also been divided by age group to allow for the sampling process (table). There were 43 certificates mentioning asthma and the panel decided death to be due to "other" causes in 29 and asthma in 14 (table). These 14 deaths had previously been registered as myocardial infarction or cardiac failure (10), cor pulmonale (1), pulmonary embolism (1), pulmonary aspergillosis (1), and poisoning with propranolol (1). Four of the 43 deaths had been incorrectly registered as cardiac failure, cor pulmonale, pulmonary aspergillosis, and pulmonary tuberculosis. There were 12 certificates mentioning COPD in cases aged under 56 and 10 were confirmed as "other" causes, one as asthma, and one as COPD. No registration errors were found in this group. There were 121 certificates mentioning COPD in cases aged $56-75$ years and death was confirmed as "other" in $88(72 \%)$, asthma in $14(12 \%)$, and COPD in $19(16 \%)$ deaths. Those confirmed as asthma had been registered as myocardial infarction (7), cardiac failure (4), stroke (1), laryngeal carcinoma (1), and diabetes mellitus (1), while those confirmed as COPD had been registered as myocardial infarction (6), cardiac failure (8), pneumonia (2), gastrointestinal haemorrhage (1), and bronchiectasis (2). In this group one certificate had been incorrectly registered as respiratory failure when COPD was the underlying cause.

Out of 350 deaths there were 11 errors in registration. In addition there was variation in the application of classification rules for both asthma and COPD. When asthma and either emphysema, COAD, or chronic bronchitis was recorded in part $\mathrm{I}$ of the certificate the death was variably registered as asthma or COPD. Of the seven cases of "asthmatic bronchitis" four were registered as asthma and three as chronic bronchitis. When COPD was recorded as being due to myocardial infarction, cardiac failure, bronchopneumonia, or pneumonia, the death was registered as COPD on 34 occasions but as the other condition on 10 occasions. In this study the number of certificates subject to variable interpretation of the classification rules where asthma or COPD without asthma was mentioned in part I of the death certificate was $13(21 \%)$ out of 62 for asthma and $44(38 \%)$ out of 117 for COPD.

In the total sample of certificates the panel diagnosed 108 deaths from asthma and 66 deaths from COPD corresponding to the 50 asthma and 124 COPD deaths registered (table). The sensitivity and specificity of death registration as an indicator of the "true" number of deaths from asthma and COPD in a subpopulation of all deaths in Northern Ireland aged up to 75 years where either asthma or COPD is mentioned anywhere on the death certificate is estimated to be $29 \%$ and $98.6 \%$ for asthma and $69 \%$ and $70 \%$ for COPD, respectively. The positive predictive value of registration of asthma deaths in this same population (the probability that a registration truly reflects an asthma death) is $87 \%$. The negative predictive value of registration of asthma deaths (the probability that a death registered under other causes was not in fact an asthma death) was $81 \%$. The corresponding figures for COPD were $39 \%$ and $88 \%$, respectively. The total number of deaths from asthma and COPD, as defined by this study, in Northern Ireland in 1987 in cases aged up to 75 is estimated to be 141 and 127, respectively. The majority of 
asthma deaths were registered under other diagnoses. Although the number of deaths from asthma has increased and from COPD has reduced, the total number of deaths from all forms of airways obstruction is similar to that registered.

The resubmission of cases before and after the relative interview caused three cases to be reassigned: the overall reproducibility of the diagnostic method was $94 \%$.

\section{Discussion}

This study has shown that death certification in asthma and COPD in Northern Ireland is inaccurate. There are many deaths currently registered as COPD which could be called asthma using currently accepted clinical diagnostic criteria.

In death certification the certifying doctor should report the cause leading directly to death on the first line of part I of the certificate and, if required, antecedent conditions on the second and third lines with the underlying cause of death stated lowest in the sequence of events. Part II of the certificate is available for listing other significant conditions contributing to the death. The registering officers restricted by the World Health Assembly rules of classification have only limited flexibility in interpreting incomplete or ambiguous certificates. ${ }^{7}$ Conditions mentioned last in part I are generally officially registered as the underlying cause of death and form the basis of published mortality statistics. Conditions mentioned in part I but with another condition mentioned beneath it on the certificate may not be registered as the underlying cause of death even when the certifying doctor intended them to be so. A condition mentioned in part II of the certificate generally cannot be registered as the underlying cause of death if a condition listed in part $I$ is a probable cause of death unless there is no doubt in the officer's mind that this condition is a direct sequel of the condition mentioned in part II - a possible or even probable causal sequence is not sufficient. Inaccuracy in the process of death certification and registration can occur if the certifying doctor misdiagnosed the cause of death, if the death certificate is incomplete or ambiguous, or if the registering officer misapplies the rules of classification. Application of some classification rules are arbitrary and can easily lead to variable registration practice by officers, especially when certificates are incomplete or ambiguous. This study has shown that there is variation in the application of the classification rules for some certificates in both asthma and COPD. The size of such variation is not insubstantial and could account for some year on year variation and geographical variation in both asthma and COPD mortality rates.

The nomenclature of asthma and other forms of chronic airways obstruction has changed in keeping with improvements in the understanding of airways obstruction. The World Health Organisation (WHO) in the International Classification of Disease 9th revision $^{7}$ included asthma in the group "chronic obstructive pulmonary disease and allied conditions", and the ICD 10th revision ${ }^{8}$ is little changed. Clinically some distinction between asthma and other forms of chronic airways obstruction appears to be useful. For this study we have made a distinction between asthma and COPD, using COPD as a collective term for all non-asthmatic chronic airways obstruction. In the UK the term chronic obstructive airways disease (COAD) is generally used for this purpose and has become increasingly used on death certificates, largely replacing chronic bronchitis. The WHO introduced the term "chronic airways obstruction unspecified" in 1978 and this is the term registered when COAD or COPD or equivalent is written on the death certificate. This study suggests that the diagnostic label of COAD written on a death certificate is often used as a convenient term for all types of chronic airways obstruction including asthma and also for other chronic respiratory conditions.

This is the first study to investigate the accuracy of death certification in all forms of airways obstruction in age groups up to 75 years. Information was obtained from all available sources. Particularly informative were details obtained from relatives who provided a lifetime clinical history unavailable from any other source.

The diagnosis of asthma and COPD retrospectively cannot be entirely objective since most deaths are not confirmed by necroscopy. The accuracy of the panel's diagnosis may be influenced in favour of asthma by bias in the diagnostic process. However, the panel consisted of two experienced respiratory physicians who diagnosed asthma or COPD as far as possible as they would in their normal clinical practice using predefined diagnostic criteria similar to those developed by the American Thoracic Society. ${ }^{6}$ In most cases they found the diagnosis to be relatively straightforward. Furthermore, the panel diagnosis of either asthma or COPD was a preliminary step of an investigation of risk factors in death from both asthma and COPD. It was not a search for asthma deaths.

Twenty deaths from asthma had been previously registered as myocardial infarction or cardiac failure. The transfer of the diagnosis to asthma was felt justified if there was no previous history of cardiac disease and if, before the "sudden death", the increase in respiratory symptoms was more in keeping with deteriorating asthma and acute respiratory failure than cardiac failure.

The inaccuracy in certification has implications for following trends in asthma and COPD mortality. The recently reported increase in asthma deaths in the elderly could be explained by relatively small shifts in death certification from COPD to asthma rather than changes in the prevalence or case fatality of asthma. In a study of death certification practice in the then European Community ${ }^{10}$ it was shown that significant variation exists in the way doctors certify a death when there is clinical ambiguity as to whether the diagnosis is asthma or COPD. In countries with high asthma mor- 
tality more doctors certified ambiguous cases as asthma than COPD. Our data show that this variation in certification could contribute to the differences in mortality rates between countries, and highlights the need for caution when interpreting international or even national regional differences.

Most investigators of asthma mortality have avoided subjects aged over 55 years and those with significant irreversible obstruction because of the difficulty in precise diagnosis. ${ }^{11}$ However, asthma mortality is high in these groups so investigation is important for a proper understanding of the risk factors involved. Moreover, it may not be possible to generalise from lessons learnt from asthma death studies in highly selected and younger age groups. It is at least possible that inaccuracy in death certification reflects poor diagnosis and management in the past.

This study confirms that death certification in asthma is inaccurate and that a similar inaccuracy occurs for COPD. Although a few errors occurred in the registration of the cause of death by the registering officers, most errors occurred on completion of the death certificate. Only by improvement in the nomenclature of obstructive airways disease, the standard of completion of death certificates, and consistent application of classification rules will mortality trends in asthma and other forms of chronic airways obstruction become interpretable.

The study was funded by the Department of Health and Social Services (NI), the Chest Heart and Stroke Association (NI), and Boehringer Ingelheim Ltd.

1 Office of Population Censuses and Surveys. Mortality statistics - cause. Series DH1-20 (1974-92). London: HMSO, 1977-94.

2 British Thoracic Association. Accuracy of death certificates in bronchial asthma. Thorax 1984;39:505-9.

3 Sears MR, Rea HH, de Boer G, Beaglehole R, Gillies AJD, Holst PE, et al. Accuracy of certification of deaths due to asthma: a national study. Am $f$ Epidemiol 1986;124: to asthma:

4 Sutherland DC, Beaglehole R, Fenwick J, Jackson RT, Mullins P, Rea HH. Death from asthma in Auckland: circumstances and validation of causes. $N Z \operatorname{Med} \mathcal{F} 1984$; 97:845-8.

5 Wright SC, Evans AE, Sinnamon DG, MacMahon J. Asthma mortality and death certification in Northern Ireland. Thorax 1994;49:141-3.

6 American Thoracic Society. Standards for the diagnosis and care of patients with chronic obstructive pulmonary disease (COPD) and asthma. Am Rev Respir Dis 1986;133:225-44.

7 World Health Organisation. International classification of disWorld Health Organisation.
eases. 9th revision, 1975.

8 World Health Organisation. International statistical classification of diseases and related health problems. 10th revision, 1992.

9 Burney PGJ. The effect of death certification practice on recorded national asthma mortality rates. Rev Epidem Sante Publ 1989;37:385-9.

10 Kelson MC, Heller RF. The effect of death certification and coding practices on observed differences in respiratory disease mortality in 8 EEC countries. Rev Epidem Sante Publ 1983;31:423-32.

11 Rea HH, Scragg R, Jackson R, Beaglehole R, Fenwick J, Sutherland DC. A case-control study of deaths from asthma. Thorax 1986;41:833-9. 